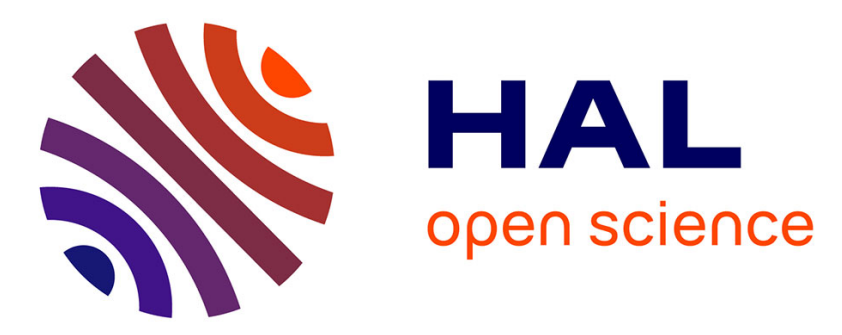

\title{
Soot Pyrometry by Emission Measurements at Different Wavelengths in Laminar Axisymmetric Flames
}

\author{
J. Cruz, Luis Fernando Figueira da Silva, F. Escudero, F. Cepeda, J.
}

Elicer-Cortés, A. Fuentes

\section{- To cite this version:}

J. Cruz, Luis Fernando Figueira da Silva, F. Escudero, F. Cepeda, J. Elicer-Cortés, et al.. Soot Pyrometry by Emission Measurements at Different Wavelengths in Laminar Axisymmetric Flames. Combustion Science and Technology, 2022, 194 (8), pp.1643-1660. 10.1080/00102202.2020.1825401. hal-03313666

\section{HAL Id: hal-03313666 \\ https://hal.science/hal-03313666}

Submitted on 8 Oct 2021

HAL is a multi-disciplinary open access archive for the deposit and dissemination of scientific research documents, whether they are published or not. The documents may come from teaching and research institutions in France or abroad, or from public or private research centers.
L'archive ouverte pluridisciplinaire HAL, est destinée au dépôt et à la diffusion de documents scientifiques de niveau recherche, publiés ou non, émanant des établissements d'enseignement et de recherche français ou étrangers, des laboratoires publics ou privés. 


\title{
Soot Pyrometry by Emission Measurements at Different Wavelengths in Laminar Axisymmetric Flames
}

\author{
J.J. Cruz ${ }^{\mathrm{a}}$, L.F. Figueira da Silva*b ${ }^{* \mathrm{~b}}$ F. Escudero ${ }^{\mathrm{a}}$, F. Cepeda ${ }^{\mathrm{a}}$, \\ J.C. Elicer-Cortés ${ }^{\mathrm{c}}$, and A. Fuentes ${ }^{\mathrm{a}}$ \\ ${ }^{a}$ Departamento de Industrias, Universidad Técnica Federico Santa \\ María, Av. España 1680, Valparaíso, Chile \\ ${ }^{\mathrm{b}}$ Department of Mechanical Engineering, Pontifícia Universidade \\ Católica do Rio de Janeiro, R. Marquês de São Vicente, 225, Rio \\ de Janeiro, Brazil \\ ${ }^{\mathrm{c}}$ Departamento de Ingeniera Mecánica, Universidad de Chile, \\ Beauchef 851, Piso 5, Santiago, Chile
}

\begin{abstract}
In this work, a multiwavelength pyrometry method is proposed in order to evaluate soot temperature for laminar axisymmetric non-premixed flames. A single radiative model that combines three detection wavelengths is developed to increase the spectral information used in the soot temperature determination. A simple set-up is used in order to capture the soot emission at two or three wavelengths. The robustness of the methodology is assessed by a radiative model, which includes a sensitivity and relative error analysis for the soot temperature, and accounts for self-absortion effects. The model and procedure are verified by employing numerical temperature and soot volume fraction fields to recreate the convoluted soot emissions. Soot emission measurements involve different combinations of wavelengths that are employed to evaluate soot temperature without requiring additional measurements of the soot absorption coefficient when soot self-absorption is neglected. The error on the determined soot temperature was estimated to be lower than $60 \mathrm{~K}$ under this assumption for soot volume fractions under $10 \mathrm{ppm}$. In addition, an analysis of the signal noise effect on the temperature shows that the modulated absorption/emission (MAE) technique is more sensitive than the emission (EMI) technique. A sensitivity analysis showed that the presented three wavelength EMI model is less sensitive to small deviations
\end{abstract}

*Corresponding author: L.F. Figueira da Silva. E-mail: luisfer@puc-rio.br; ORCID: 00000002-4932-7341 
on the measured soot emission ratio than the classical two wavelength approach. This feature makes the model suitable to improve the accuracy on the determination of soot temperature when the noise level of the signal is significant.

keywords: Three color pyrometry, coflow flame, self-absorption, soot temperature, error analysis. 


\section{Introduction}

Temperature is one of the most important thermodynamic properties to be determined in combustion processes studies. Indeed, the reacting mixture temperature exerts a controlling influence on the chemical reaction rate, which is classically modelled using an Arrhenius-type expression for gas phase kinetics. Such a non-linear dependency, together with the high activation energies typically found, implies that minute temperature determination errors translate into large reaction rate uncertainties. This also explains why temperature is often used as a basis for comparisons between numerical modelling and experimental results (Andrade et al. 2011, Vedovoto et al. 2015, Jerez et al. 2019). Therefore, accurately measuring the temperature field is paramount to the development of novel combustion models, in particular when poorly known chemical reaction pathways are of interest, which is the case of soot or particle formation in flames (Wang 2011).

Soot represents agglomerates of small carbonaceous particles, with diameters that lie between 5 and $60 \mathrm{~nm}$ (Hadef et al. 2013, Patiño et al. 2020), formed by the incomplete fuel burning. The soot radiation emission from flames presents spectral characteristics that have been studied for decades. Indeed, soot particles emit a broadband spectral radiation in the visible (Migliorini et al. 2011) due to their carbonaceous nature, for typical flame temperatures. Therefore, the spectroscopic analysis of soot radiation at spectral regions free of other species interference has often been used to determinate its volume fraction distribution, temperature and radiative properties (Zhao and Ladommatos 1998, Araujo 2017).

When thermal radiation is transported throughout a flame, soot emission may be absorbed by the adjacent participating medium. Considering the classical particle cloud model, the exponential term that describes this self-absorption process poses an important problem to the solution of the associated integral equation (Liu et al. 2013). This solution requires the knowledge of the soot absorption coefficient, $k_{\lambda}$, its determination being subject of several proposed techniques. For instance, the Modulated Absorption/Emission technique (MAE) (Jenkins and Hanson 2001, Legros et al. 2015) relies on light extinction measurements to determine this coefficient. The multi-spectral soot emission technique (De Iuliis et al. 1998, Snelling et al. 2002) determines the soot temperature via a curve fitting between the measured emission and its corresponding detection wavelengths. These approaches require external light sources (e.g. a laser) or careful independent spectrograph calibration at several detection bands, which could constitute a source of uncertainties. These uncertainties are over-layered to other error sources, such as the soot refractive index function value (Goulay et al. 2009) and the experimental noise ( $\mathrm{Li}$ and $\mathrm{He}$ 2019). Different methods have been proposed to solve the integral equation when the self-absorption term is not accounted for, but which correct the attenuated measured signals by modelling the projected signal ratio without and with self-absorption (Snelling et al. 2002, Freeman and Katz 1960). However, these methods also require the knowledge of soot properties, such as the absorption 
coefficient $\left(k_{\lambda}\right)$. The ratio color pyrometry technique involves a function that relates the soot temperature with the ratio of soot emission measured at different wavelengths (Jenkins and Hanson 2001, Legros et al. 2015, Panagiotou et al. 1996, Jakob et al. 2014, Escudero et al. (2016). In cases where self-absorption is neglected, the radiative model is simplified to an Abel integral equation. Errors of around tenths of Kelvin have been reported (Liu et al. 2013. Kempema and Long 2018) when the ratio of two wavelengths emission is used.

Even if more advanced soot measurement techniques are available, emission pyrometry remains a viable technique when the optical access to the flame is limited, which is the case of several industrial processes, such as oil refining furnaces. Therefore, the accurate characterization - and subsequent minimization - of the sources of errors could benefit the end-user in those industrial processes.

The objective of this work is to present an improved three-color based methodology to determine soot temperature without the explicit knowledge of the soot absorption coefficient. This methodology is based on the measurement of soot emission at different detection wavelength combinations, while neglecting the radiation self-absorption effect. A brief description of the experimental setup is first given. Then, the radiative model used to determine the soot temperature is presented, as well as the corresponding sensitivity and relative error analysis of the three wavelengths pyrometry. The robustness of the methodology is numerically evaluated, as is the inherent error resulting from the assumption of an optically thin flame. The different experimental temperature results are compared with the numerical model counterpart, and finally the main conclusions are drawn.

\section{Experimental procedure}

This work uses a simple set-up based in Emission Measurements (EMI), which is depicted in Fig. 1, to evaluate the soot temperature at different wavelengths. The EMI method set-up is considerably simpler than other soot pyrometry techniques, such as those based on combined emission and attenuation. Indeed, the optical arrangement required to capture the emission and attenuation signals, which has been applied to laminar 1-D flames and common laminar 2-D axisymmetrical configurations (Jenkins and Hanson 2001, Legros et al. 2015), is rather difficult to adapt to industrial applications. In these set-ups two light sources are required at different wavelengths in order determine the light absorption and simultaneously capture the soot emission. These signals are measured at the same wavelengths by a photodetector or a camera, pertinently triggered. Thus, the extinction system involves additional complications, such as the need for sample

optical access, the alignment between laser and camera and synchronization of several devices, which hinder the practical implementation for industrial applications. On other hand, on the employed set-up, only the collection of soot emissions is required. This practical optical arrangement allows to capture the soot emission at several wavelengths straightforwardly. The reliability of the EMI methodology has recently been demonstrated for two wavelengths (Escud- 

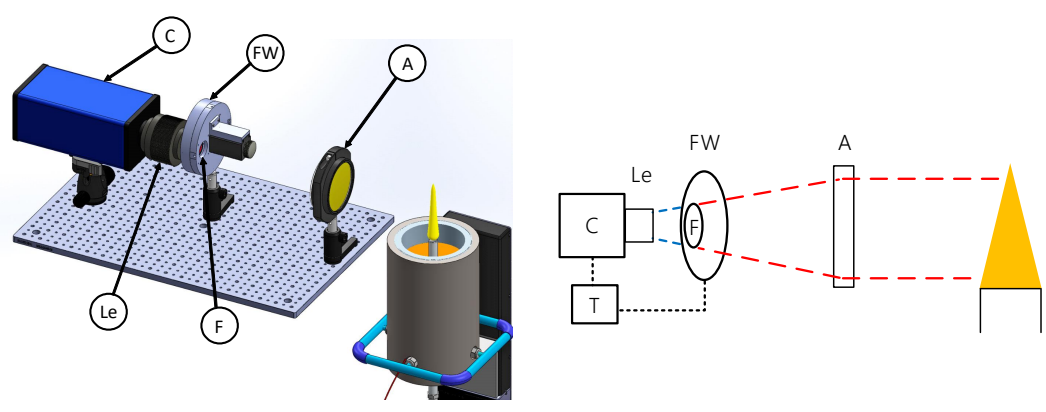

Figure 1: Scheme of the telecentric optical configuration for the Emission Measurements (EMI); A: Achromatic, C: CCD or CMOS Camera, F: Filters, FW: Motorized filter wheel, Le: Lenses, T: Trigger controller.

ero et al. 2016) and this method is applied here for three wavelengths, thus demonstrating the robustness of the soot pyrometry technique proposed by using EMI only.

The EMI optical configuration requires the devices presented in Fig. 1 only. In order to collect the thermal radiation emitted by the soot within the flame, the set-up incorporates an EO 88-598 achromatic lens (A) of focal distance $f=400 \mathrm{~mm}$ and $75 \mathrm{~mm}$ diameter $(400-1000 \mathrm{~nm})$. The EMI signal is filtered (F) at the desired wavelength by means of a Thorlabs motorized filter wheel (FW) model FW103H/M, which guarantees a fast spectral detection band selection. Three narrow-band filters (F, Thorlabs) are installed at the motorized FW. The radical species (Zhou et al. 2017) and $C_{2}$ clusters emissions are avoided by the selected filters (Goulay et al. 2010 ). These filters (F) are centered at wavelengths of $\lambda_{1}=532 \pm 5 \mathrm{~nm}, \lambda_{2}=660 \pm 5 \mathrm{~nm}$ and $\lambda_{3}=800 \pm 5 \mathrm{~nm}$, thus allowing to detect the soot emission only. A Navitar $f 2.8 / 50 \mathrm{~mm}$ lens (Le) is coupled to an Andor Luca R CCD camera (C) with $1004 \times 1002$ pixels and 14 bit depth, which allows to capture flame emission images with a spatial resolution of $17 \mathrm{px} / \mathrm{mm}$. The collection arrangement represents a telecentric configuration (Greenberg and $\mathrm{Ku}$ 1997), where a small objective iris ( $f / 11)$ aperture and 400 $\mathrm{mm}$ of separation between the achromatic lens and the burner have been chosen in order to improve the collection of the parallel light rays emitted by the flame. The CCD camera and the motorized FW are externally synchronized during EMI measurements. The EMI methodology is applied to a 2-D laminar axisymmetrical non-premixed flame stabilized in a Gülder-type burner (Snelling et al. 1999), configuration that is commonly employed to study soot formation/oxidation processes (Escudero et al. 2016, Jerez et al. 2019). This burner consists of a $10.9 \mathrm{~mm}$ internal diameter stainless steel fuel tube and a larger concentric tube of $90 \mathrm{~mm}$ internal diameter devoted to the oxidizer coflow. Ethylene is injected at a fixed flow rate of $0.194 \mathrm{slpm}$, whereas the air flow stream is supplied at $284 \mathrm{slpm}$. Two flow controllers Brooks MFC are employed to deliver the gaseous fuel and the air (models SLA5850S and SLA5853S, respectively). 


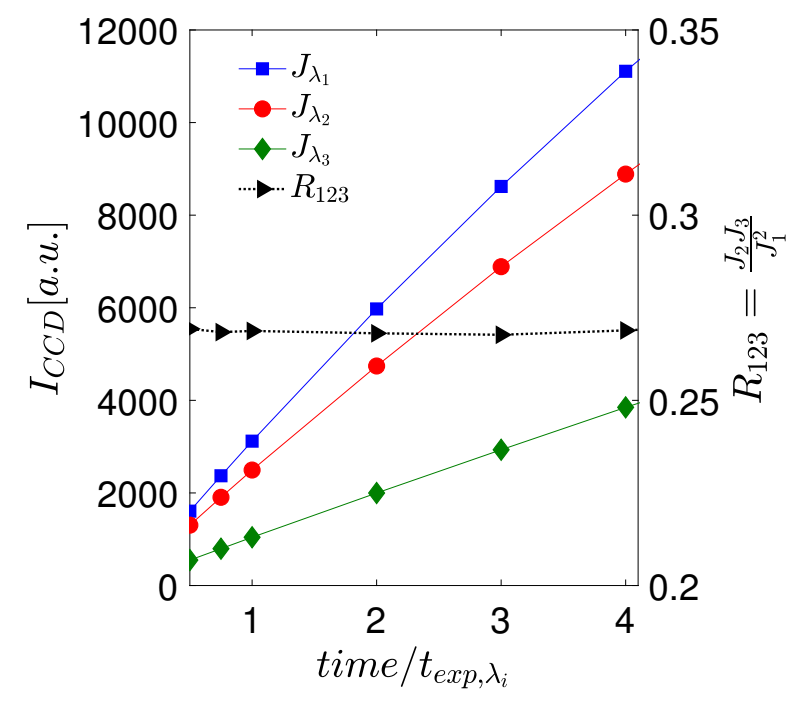

Figure 2: Signal intensity and $R_{123}$ as a function of the exposure time ratio at each detection wavelength, where $t_{\exp , 532}=0.055 \mathrm{~s}, t_{\exp , 660}=0.016 \mathrm{~s}$ and $t_{\text {exp }, 800}=0.01 \mathrm{~s}$.

During the experiments, 150 images are sequentially measured at the detection wavelengths $\lambda=532,660,800 \mathrm{~nm}$, using exposure times of $t_{\text {exp }, \lambda}=0.055$, 0.016 and $0.01 \mathrm{~s}$, respectively. These times have been chosen in order to maximize the flame signal detection at each wavelength. In order to verify the optical system linear response, radiation measurements of a tungsten lamp (Thorlabs SLS202L/M), at constant power, have been performed with different exposure times. Figure 2 shows that the detection system response remains linear when the exposure time at each wavelength, $t_{\exp , \lambda_{i}}$, is multiplied by factors ranging from 0.5 to 4 . This figure also shows that the $R_{123}=\left(J_{\lambda_{2}} J_{\lambda_{3}}\right) / J_{\lambda_{1}}^{2}$ relation remains nearly constant when the signal, $J_{\lambda_{i}}$, proportionality is linear.

The global transmissivity of the detection system, including the achromatic and band-pass filters, lens and CCD response, has been evaluated by comparing the camera signals at each detection wavelength by imaging a calibrated tungsten light source (Thorlabs SLS202L/M). The calculated spectral factors have been used to correct the camera images intensities and, thus, to retrieve the flame spectral emission. Each group of images is averaged and corrected accounting for the calibration factor at each detection wavelength. The resulting EMI images represent the projected line-of-sight soot emission, which are Abel-inverted using an onion-peeling technique together with a Tikhonov regularization procedure (Daun et al. 2006, Escudero et al. 2016) in order to obtain the local soot emission at different wavelengths. 


\section{Radiative model for soot pyrometry}

If the flame radiation measurements are carried out at spectral regions where the soot emission dominates the flame spectrum, the measured signal intensity represents the soot emission integrated along the line-of-sight. However, soot radiation emitted within the flame may be attenuated by absorption and scattering of adjacent soot particles before reaching the photo-detector. In the present study it is assumed that the soot particles characteristic dimensions are small when compared to the detection wavelength. As a consequence, the radiation/particle interaction should occur within the Rayleigh regime (Dobbins and Megaridis 1991), thus allowing to neglect light scattering effects. Given these considerations, the flame behaves as an emitting and absorbing medium (Liu, Thomson and Smallwood 2009) and the soot emission, $I_{\lambda}$, along the optical path $l$, at wavelength $\lambda$, may be modeled by the radiative transfer equation (RTE) (Viskanta and Mengüç 1987):

$$
\frac{d I_{\lambda}}{d l}=J_{\lambda}(l)-k_{\lambda} I_{\lambda},
$$

where $k_{\lambda}$ represents the soot absorption coefficient, and the spectral soot emission is expressed as $J_{\lambda}=k_{\lambda} I_{b \lambda}$, with hypothesis of local thermodynamic equilibrium (LTE) and Kirchhoff's law (Modest 2013). The monochromatic blackbody radiative intensity at temperature $T$ per unit area and unit solid angle, $I_{b \lambda}$, is given by Planck's law. The integrated solution of Eq. (1), along the photo-detector line-of-sight $(y)$ is given by:

$$
I_{\lambda}(y)=\int_{l_{0}(y)}^{l_{f}(y)} J_{\lambda}(l) \exp \left[-\int_{l}^{l_{f}(y)} k_{\lambda}\left(l^{\prime}\right) \mathrm{d} l^{\prime}\right] \mathrm{d} l,
$$

where $l_{0}(y)$ and $l_{f}(y)$ represent the optical flame path limits, and the exponential term describes the soot self-absorption. Previous studies have shown that self-absorption could be important for flames with large optical thickness, and neglected when the optical thickness is smaller than 0.3 (Liu et al. 2013).

In the present work the soot temperature is measured considering the flame to be optically thin, nevertheless the self-absorption effect is also included in the model and analyzed. The modified Abel integral Eq. (2) must be deconvoluted, so as to obtain the local soot emission intensity as a function of the radial coordinate $r, J_{\lambda}(r)=k_{\lambda}(r) I_{b \lambda}(r)$.

The experimental measurements capture the soot emission at a spectral band $\lambda \pm \delta \lambda$. Considering the signal measured by the CCD sensor to be proportional to $I_{\lambda}$ (see Eq. (2)) and that the flame is optically thin, one may write:

$$
I_{\lambda, C C D}(y) \propto C \int_{\lambda-\delta \lambda / 2}^{\lambda+\delta \lambda / 2} \eta_{\lambda} D_{\lambda}\left[\int_{l_{0}(y)}^{l_{f}(y)} k_{\lambda} I_{b \lambda}, \mathrm{d} l\right] \mathrm{d} \lambda .
$$

In Eq. 3. $\eta_{\lambda}$ is the transmissivity of the optical arrangement (neutral density filter, lens and filters), $D_{\lambda}$ is the spectral response of the CCD, and $C$ involves 
the photo-detector exposure time and geometrical factors of optical setup, that are globally determined by using a calibration light source. A tomographic deconvolution procedure, developed by (Legros et al. 2015, Escudero et al. 2016) has been employed to solve the Eq. (2) and Eq. (3). This procedure consist in an onion-peeling algorithm, together with a Tikhonov regularization technique (Daun et al. 2006).

Once the local soot intensity fields $\left[J_{\lambda_{i}}(r)\right]$ are calculated by deconvolution, the intensity ratio at two wavelengths $\lambda_{1}<\lambda_{2}$, may be obtained as:

$$
R_{12}=\frac{J_{\lambda_{1}}(r)}{J_{\lambda_{2}}(r)}=\frac{k_{\lambda_{1}}(r)}{k_{\lambda_{2}}(r)} \frac{c_{1} / \lambda_{1}^{5}\left[\exp \left(c_{2} / \lambda_{1} T\right)-1\right]}{c_{1} / \lambda_{2}^{5}\left[\exp \left(c_{2} / \lambda_{2} T\right)-1\right]} .
$$

An explicit solution to the soot temperature is obtained using Wien's approximation of Planck's law, in the $\lambda T<3000 \mu \mathrm{m}$ range (Di Stasio and Massoli 1994), which is valid for the detection ranges used in this work. Therefore, the soot temperature is given by (Legros et al. 2015):

$$
T_{12, \text { MAE }}=\frac{C_{2}\left(\frac{1}{\lambda_{2}}-\frac{1}{\lambda_{1}}\right)}{\ln \left[R_{12} \frac{k_{\lambda_{2}}}{k_{\lambda_{1}}}\left(\frac{\lambda_{1}}{\lambda_{2}}\right)^{5}\right]},
$$

This equation is used in the MAE technique and requires knowledge of the soot absorption coefficient $\left(k_{\lambda}\right)$, that is typically obtained by light extinction measurements with an external light source. The $k_{\lambda}$ field is retrieved by deconvolution of the medium transmissivity $\left(\tau_{\lambda}\right)$, as $\tau_{\lambda}=\exp \left(-\int k_{\lambda} \mathrm{d} l\right)$.

In the Rayleigh regime, the soot absorption coefficient, $k_{\lambda}$, is related to the soot volume fraction, $f_{v}$, via $k_{\lambda}=f_{v} C_{\lambda} / \lambda$ (Modest 2013). As $f_{v}$ is a spectrally independent physical property, Eq. (5) may be rewritten in terms of soot emission only, by adopting a model for the soot absorption function $E(m)$ in $C_{\lambda}=6 \pi E(m)$, given by (Jenkins and Hanson 2001):

$$
T_{12, E M I}=\frac{C_{2}\left(\frac{1}{\lambda_{2}}-\frac{1}{\lambda_{1}}\right)}{\ln \left[R_{12} \frac{C_{\lambda_{2}}}{C_{\lambda_{1}}}\left(\frac{\lambda_{1}}{\lambda_{2}}\right)^{6}\right]} .
$$

The procedure to obtain $T_{13}$ or $T_{23}$ is identical, and the indices should be modified accordingly. In this work, when the EMI method is used, the soot selfabsorption is not considered in the solution of Eq. (3). Note that using the calculated $T$ and $J_{\lambda}$ fields together with the $E(m)$ model, it is also possible to determine the soot volume fraction, which has not been performed for the sake of brevity. In addition, the knowledge of the absolute $E(m)$ value is required to determine the soot volume fraction, whereas to determine the soot temperature, relative spectral variation of $E(m)$ with the wavelength is sufficient (Snelling et al. (2002).

However, the $E(m)$ models reported in the literature are an issue of ongoing debate. Indeed, $E(m)$ has been shown to exhibit either an increasing (Michelsen 
et al. 2010) or decreasing trend (Yon et al. 2011) within the visible spectral region. Here the light scattering by the soot particles is assumed to be negligible, and the model proposed by Chang and Charalampopoulos (1990) is adopted to determine the $E(m)$ value. This is motivated by the need to maintain consistency with the $f_{v}$ and $T$ fields numerically computed by the CoFlame code (Eaves et al. 2016), and that are used here to compute a numerical local soot emission, $J_{\lambda}$ for the validation of this methodology (see Section 5.1).

Concerning the three color soot pyrometry technique, with $\lambda_{1}<\lambda_{2}<\lambda_{3}$, two ratios may be derived from Eq. (4) (Khan et al. 1991):

$$
R_{123}=R_{12} \cdot R_{13}=\frac{\left[J_{\lambda_{1}}(r)\right]^{2}}{J_{\lambda_{2}}(r) J_{\lambda_{3}}(r)} .
$$

Then, the temperature for the three wavelengths, $T_{123}$, with the MAE method is obtained following:

$$
T_{123, M A E}=\frac{C_{2}\left[\left(\frac{1}{\lambda_{2}}-\frac{1}{\lambda_{1}}\right)+\left(\frac{1}{\lambda_{3}}-\frac{1}{\lambda_{1}}\right)\right]}{\ln \left\{R_{123} \frac{k_{\lambda_{2}} k_{\lambda_{3}}}{\left(k_{\lambda_{1}}\right)^{2}}\left[\frac{\left(\lambda_{1}\right)^{2}}{\lambda_{2} \lambda_{3}}\right]^{5}\right\}},
$$

whereas for case of the EMI method one may write:

$$
T_{123, E M I}=\frac{C_{2}\left[\left(\frac{1}{\lambda_{2}}-\frac{1}{\lambda_{1}}\right)+\left(\frac{1}{\lambda_{3}}-\frac{1}{\lambda_{1}}\right)\right]}{\ln \left\{R_{123} \frac{C_{\lambda_{2}} C_{\lambda_{3}}}{\left(C_{\lambda_{1}}\right)^{2}}\left[\frac{\left(\lambda_{1}\right)^{2}}{\lambda_{2} \lambda_{3}}\right]^{6}\right\}} .
$$

\section{Sensitivity analysis and relative error}

The relative error analysis for soot temperature $\left(T_{123}\right)$ from the measured emission, $J_{\lambda_{i}}$, and the soot emission ratio, $R_{123}$, is performed by taking the partial derivative of the temperature (Eq. (9p) with respect to these quantities, thus obtaining:

$$
\begin{aligned}
R_{123} \frac{\partial T}{\partial R_{123}} & =\frac{1}{2} J_{\lambda_{1}} \frac{\partial T}{\partial J_{\lambda_{1}}}=-J_{\lambda_{2}} \frac{\partial T}{\partial J_{\lambda_{2}}}=-J_{\lambda_{3}} \frac{\partial T}{\partial J_{\lambda_{3}}} \\
& =\frac{T^{2} \lambda_{1} \lambda_{2} \lambda_{3}}{C_{2}\left[\lambda_{3}\left(\lambda_{2}-\lambda_{1}\right)+\lambda_{2}\left(\lambda_{3}-\lambda_{1}\right)\right]} .
\end{aligned}
$$

Eq. 10 allows different approaches to evaluate the temperature response to small changes in $J_{\lambda_{i}}$. Also, it is possible to deduce the relative sensitivity of temperature $(\delta T / T)$ with respect to the relative signal ratio $\left(\delta R_{123} / R_{123}\right)$ and signal emissions $\left(\delta J_{\lambda_{i}} / J_{\lambda_{i}}\right)$ as:

$$
\begin{aligned}
\frac{\partial \ln T}{\partial \ln R_{123}} & =\frac{1}{2} \frac{\partial \ln T}{\partial \ln J_{\lambda_{1}}}=-\frac{\partial \ln T}{\partial \ln J_{\lambda_{2}}}=-\frac{\partial \ln T}{\partial \ln J_{\lambda_{3}}} \\
& =T \frac{\lambda_{1} \lambda_{2} \lambda_{3}}{C_{2}\left[\lambda_{3}\left(\lambda_{2}-\lambda_{1}\right)+\lambda_{2}\left(\lambda_{3}-\lambda_{1}\right)\right]} .
\end{aligned}
$$


These ratios indicate that the relative deviations depend on the calculated soot temperature and separation between the wavelengths employed only. Therefore, the three wavelengths soot temperature [Eq. (9)] relative errors may be obtained using:

$$
\begin{gathered}
\frac{\partial T}{\partial \lambda_{1}}=\frac{2 T \lambda_{2} \lambda_{3}\left(C_{2}-6 \lambda_{1} T\right)}{\lambda_{1} C_{2}\left[\lambda_{3}\left(\lambda_{1}-\lambda_{2}\right)+\lambda_{2}\left(\lambda_{1}-\lambda_{3}\right)\right]}, \\
\frac{\partial T}{\partial \lambda_{j}}=\frac{-T \lambda_{1} \lambda_{k}\left(C_{2}-6 \lambda_{j} T\right)}{\lambda_{j} C_{2}\left[\lambda_{3}\left(\lambda_{1}-\lambda_{2}\right)+\lambda_{2}\left(\lambda_{1}-\lambda_{3}\right)\right]}, \quad\{j, k\} \in\{2,3\},
\end{gathered}
$$

and the sensitivity of $\ln T$ to $\ln \lambda_{i}$ may be written as:

$$
\begin{gathered}
\frac{\partial \ln T}{\partial \ln \lambda_{1}}=\frac{2 \lambda_{2} \lambda_{3}\left(C_{2}-6 \lambda_{1} T\right)}{C_{2}\left[\lambda_{3}\left(\lambda_{1}-\lambda_{2}\right)+\lambda_{2}\left(\lambda_{1}-\lambda_{3}\right)\right]}, \\
\frac{\partial \ln T}{\partial \ln \lambda_{j}}=\frac{-\lambda_{1} \lambda_{k}\left(C_{2}-6 \lambda_{j} T\right)}{C_{2}\left[\lambda_{3}\left(\lambda_{1}-\lambda_{2}\right)+\lambda_{2}\left(\lambda_{1}-\lambda_{3}\right)\right]}, \quad\{j, k\} \in\{2,3\} .
\end{gathered}
$$

These relations may be used to provide insights on the effects of $R_{123}, J_{\lambda_{i}}$ and $\lambda_{i}$ on the measured soot temperature.

\section{$5 \quad$ Results and discussion}

This section first discusses the pyrometry technique validation by using numerical simulation data. The soot self-absorption influence on the retrieved temperature is investigated. Then, an error assessment is performed, which involves randomly perturbing the emission fields and increasing the soot volume fraction. Finally, the experimentally determined soot temperature values are compared.

\subsection{Method validation}

In order to validate the EMI methodology, simulated results of a classical target laminar ethylene/air diffusion flame are employed as input data for the purpose of testing the above described methodology. Accordingly, the soot volume fraction and temperature fields of the canonical non-smoking axisymmetric Gülder flame (Snelling et al. 1999) have been obtained from a converged solution using the CoFlame code Eaves et al. (2016). The first validation step consists on testing the deconvolution procedure applied to Eq. (2), in order to determine the local soot intensity emission $J_{\lambda}(r)$ from numerically constructed projected fields of $I_{\lambda}$, and to retrieve the original temperature field. Accordingly, Figs. 3a and $3 \mathrm{~b}$ show the computed soot volume fraction and flame temperature fields, with the solid line in Fig. $3 \mathrm{~b}$ bounding the region within where the soot concentration is $f_{v}>0.5 \mathrm{ppm}$. The maximum temperature value, $\approx 2100 \mathrm{~K}$, is found to be located at the external boundary of this sooting region. The soot emission computations are carried out within this region only, in order to be comparable with the actual experiments, where the soot emission is measured. 


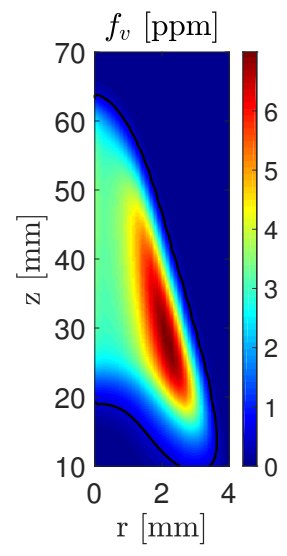

(a)

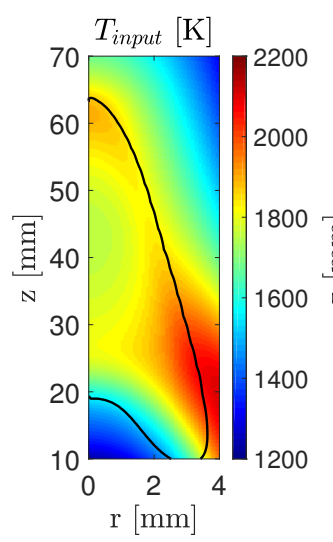

(b)

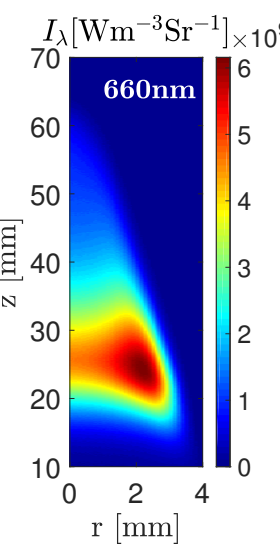

(c)

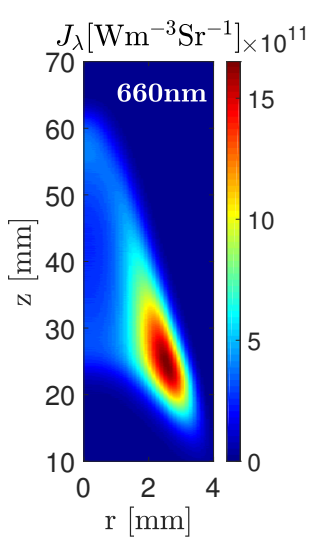

(d)

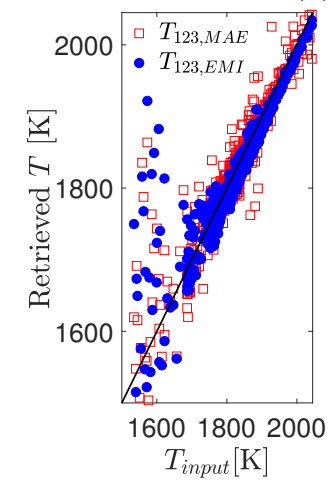

(e)

Figure 3: Numerical fields used to test the EMI methodology for an ethylene/air coflow non-premixed flame. (a) soot volume fraction $\left(f_{v}\right),(\mathrm{b})$

flame temperature $(T)$ with the iso-contour value of $f_{v}=0.5 \mathrm{ppm},(\mathrm{c})$ calculated projected integrated intensity emission $\left(I_{\lambda}\right)$ deduced from Eq. (2), (d) retrieved soot emission $\left(J_{\lambda}\right)$ after the deconvolution procedure and (e) correlation between numerical and retrieved soot temperature for three color $T_{123, M A E}$ and $T_{123, E M I}$ methods. 
The numerical $T$ and $f_{v}$ fields are employed as input values to the Planck's equation and local absorption coefficient expression, so as to determine the numerical local fields of $J_{\lambda, \text { num }}(r)=k_{\lambda}(r) I_{b \lambda}(r)$ at the flame symmetry plane. The projected soot emission field $\left(I_{\lambda, C C D}\right)$, as shown in Fig. $3 \mathrm{c}$. has been constructed by integration of the local emission field along the optical detection path at $\lambda_{2}=660 \mathrm{mn}$, following Eq. (3), but considering the self-absorption effect. It is possible to observe in Fig. 3c that the zone of large $I_{\lambda}$ is associated with significant soot concentrations. This figure also shows that, at the zone close to the burner exit $(z<20 \mathrm{~mm})$, the flame exhibits low temperature and $f_{v}$ values, thus leading to a zone of smaller $I_{\lambda}$ values. Also, in this region the flame is thicker, thus underscoring the soot emission attenuation.

In order to emulate the usual random errors encountered in experiments, an uncorrelated numerical noise is introduced in the projected intensity field $I_{\lambda}$ scaled to the camera dynamic range, which should thus represent the common measured soot emission intensities captured with a CCD or CMOS camera. To this end, a statistical white noise with zero mean and a standard deviation of $5 \%$ of the mean camera background noise $(500$ a.u.) is added to the three $\left(\lambda_{1}, \lambda_{2}, \lambda_{3}\right)$ numerically integrated fields. Figure 3d presents the retrieved local soot emission field, $J_{\lambda, r e t v}(r)$, obtained from deconvolution of $I_{\lambda}$ with a Tikhonov regularization parameter of $\alpha_{r e g}=10^{-5}$. For the MAE method, the fields of $\tau_{\lambda}$ used to consider the self-absorption were altered by adding white noise of zero mean and standard deviation equal to $0.25 \%$ of the minimum value of $\tau_{\lambda}$.

Replacing the retrieved intensities $\left[J_{\lambda, \text { retv }}(r)\right]$ ratios in Eqs. (6) and (9), it is possible to determie the corresponding retrieved temperature fields. Figure $3 \mathrm{e}$ gives the correlation between the original and the retrieved soot temperature values using the models for three wavelengths. An excellent agreement may be observed above $1500 \mathrm{~K}$, regardless the soot temperature model used, demonstrating the robustness of the adopted procedure. This figure also shows that discrepancies of up to $150 \mathrm{~K}$ appear along the flame for temperatures below $1500 \mathrm{~K}$ when the white noise is introduced. These discrepancies, which are observed for the model and that are independent of the wavelengths employed, arise because the deviations induced by the noise are not fully compensated by the regularization procedure (Âkesson and Daun 2008) at the regions with low soot volume fraction and temperature. It is important to stress that the method is very sensitive to the ratio $R$ [Eqs. (4) and (7)] and, therefore, to the noise introduced in $I_{\lambda}$. This sensitivity should grow with the number of terms in the ratio $R$, thus leading to increased deviations for values computed with three wavelengths, when compared to the two wavelengths method (Khan et al. 1991).

\subsection{Soot self-absorption influence}

Another important aspect is to analyze the self-absorption effect induced by the soot particles on the temperature reconstruction. Indeed, the simplified methodology requires a priori knowledge of $k_{\lambda}$, as seen in Eq. (2), because 


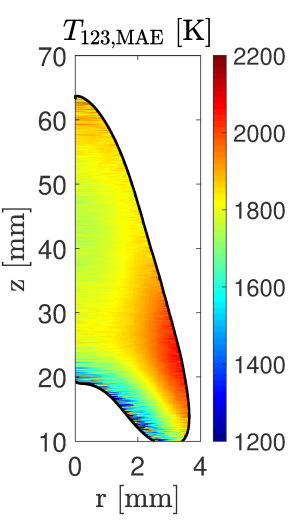

(a)

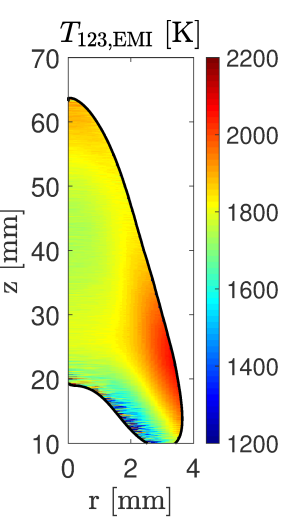

(b)

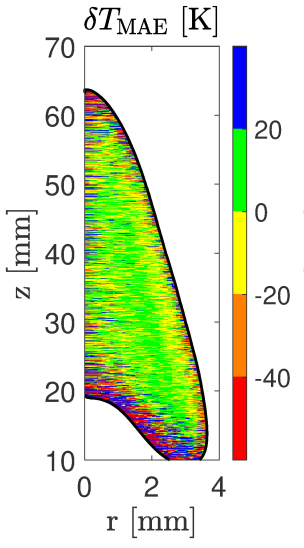

(c)

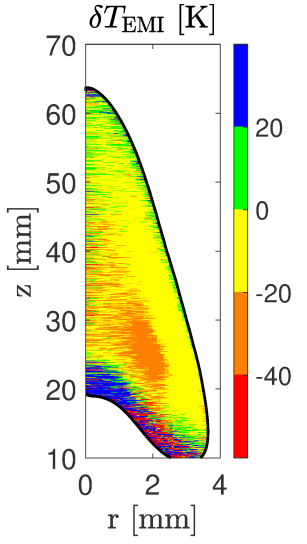

(d)

Figure 4: Soot temperature fields obtained by three color pyrometry: (a) considering the soot self-absorption effect, (b) when self-absorption is neglected, (c), (d) corresponding difference between the numerically retrieved and actual temperature distributions.

emission could be attenuated along the optical path. The proposal here is to avoid the simultaneous measurement of $f_{v}$ and temperature, by quantitatively determining the error introduced in the soot temperature value by the EMI technique when the soot self-absorption is neglected. However, if the soot selfabsorption effect is to be considered in the soot emission signal, it is necessary to determine the extinction coefficient $k_{\lambda}$, by measurements performed with the MAE technique, for instance. Note, however, that the soot influence could be simplified depending on the flame optical thickness of the (Liu et al. 2013).

Figure 4 presents the results of numerically retrieved temperature for the three wavelengths model - note that a similar behavior is observed for the two wavelengths model, not shown here for the sake of brevity. The temperature distribution is given in Fig. 4a when self-absorption is considered, and that neglecting the attenuation is depicted at Fig. $4 \mathrm{~b}$. Regular fields may be observed for locations where $T>1600 \mathrm{~K}$ and, as it could have been expected from the corresponding smaller $T$ and $f_{v}$ values, while inconsistent results are mainly obtained at the sooting region boundaries. The added white noise alters the signal-to-noise level of the projected field, further compromising the deconvolution procedure results at the region with relatively small $f_{v}$ and $T$ values.

As a manner to evidence a confidence region, the difference between the original numerical (see Fig. 3b) and the retrieved temperature values, $\delta T$, are also plotted in Figs. $4 \mathrm{c}$ and $4 \mathrm{~d}$, with and without soot self-absortion, respectively. It is possible to note a discrepancy between the two approaches by observing the limited zone with a maximum error of $30 \mathrm{~K}$ at the maximum $f_{v}$ zone (blue region in Fig. $4 \mathrm{~d}$ ), when the flame is considered optically thin. The $\delta T$ fields show that, when self-absorption is not accounted for, temperature is mainly underestimated 


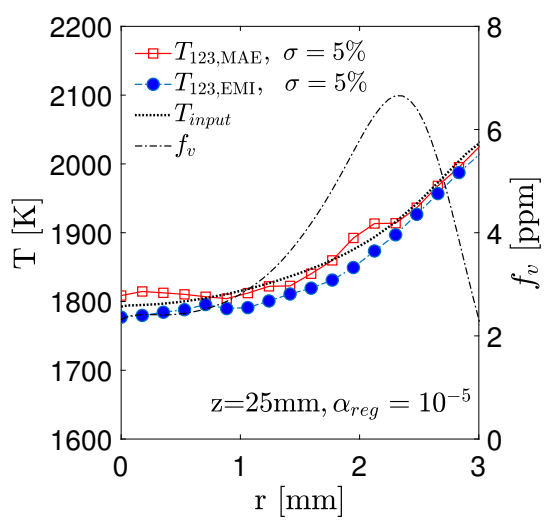

(a)

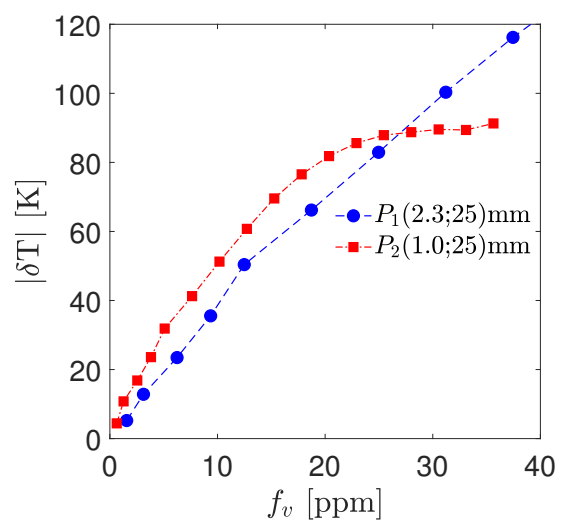

(b)

Figure 5: Influence of self-absortion on (a) the radial profiles of the retrieved three color temperature at $z=25 \mathrm{~mm}$, and (b) the absolute difference between the retrieved and actual numerical soot temperature as a function of $f_{v}$.

in zones with high soot concentration, such as for $r=2 \mathrm{~mm}$ and $z=30 \mathrm{~mm}$. As will be discussed in Section 5.3, the large irregularities observed at the flame axis are associated to the error accumulation in the deconvolution process, which are not overcome by the regularization procedure. Larger discrepancies may be observed at the sooting zone limits, which are associated to the local emission being closer to the camera background intensity. Furthermore, the annular soot distribution at the flame base causes reconstructed temperature irregularities (see Fig. 4c and 4d) as reported by Liu et al. (2013) and Wang et al. (2019). These fields allow to define a confidence region (CR), where the discrepancy of the retrieved soot temperature is sufficiently small. Therefore, based on these results and on the $f_{v}$ distribution at this target flame, it is adopted here a CR delimited by $\delta T_{123}=30 \mathrm{~K}$, i.e., $1.5 \%$ of the maximum temperature. This value is also adopted for the soot pyrometry measurement with two wavelengths.

An assessment of the $f_{v}$ influence on the soot temperature is presented in Fig. 5 a, that exhibits profiles of $T$ and $f_{v}$ for a height above the burner of $z=25 \mathrm{~mm}$. This figure allows to observe that the temperature trends both with and without self-absorption are similar, i.e, a slightly underestimation may be seen when the flame is considered optically thin, even around the $f_{v}$ peak $(\approx 6.7 \mathrm{ppm})$.

In order to further evidence the effect of self-absorption on the retrieved temperature, the values of $f_{v}$ are arbitrarily multiplied by a constant, while maintaining an identical temperature field. Figure 5b shows the absolute discrepancy, $|\delta T|$, for two radial positions, i.e., the maximum $f_{v}$ point $\left(P_{1}\right)$ and close to centerline $\left(P_{2}\right)$. The obtained results shown that $|\delta T|$ monotonically increases with $f_{v}$, reaching deviations around 100-120 K when the soot volume 
fraction is artificially increased to $40 \mathrm{ppm}$. However, the temperature discrepancies are smaller than $70 \mathrm{~K}$ when $f_{v}<15 \mathrm{ppm}$. Furthermore, for $f_{v}<10 \mathrm{ppm}$, $|\delta T|<50 \mathrm{~K}$, thus establishing another boundary for the confidence region (CR).

\subsection{Method error assessment}

The performance of the two temperature evaluation methods, MAE [Eq. (8)] and EMI [Eq. (9)], is conditional to the experimental data quality and reliability. Indeed, each image captured by the detection system represents the sum of the soot emission and the inherent experimental errors or noise. Therefore, in order to evidence the effect of random errors, the white noise level $(5 \%)$ has been artificially increased to $10 \%$ and $20 \%$ of the background intensity, and then added to each projected field, $I_{\lambda}$. Likewise, the noise of $\tau_{\lambda}$ was increased in similar proportion ( $\mathrm{x} 2$ and $\mathrm{x} 4$ ). The influence of the Tikhonov regularization parameter on the deconvolution is studied for $\alpha_{\text {reg }}=10^{-6}, 10^{-5}$ and $10^{-4}$, as show in Fig. 6a, 6b and 6c, respectively.

For a given regularization parameter, Fig. 6 shows that the temperature reconstruction performance deteriorates due to the random noise increase, for both MAE and EMI expressions. As it could have been expected, an increase on the regularization parameter improves the temperature profile reconstruction. However, the MAE results always seem to exhibit a larger scatter, regardless of the regularization value used. This may be attributed to double use of the absorption coefficient fields in Eqs. (2) and (8), where the noise difficulties the deconvolution process and increases the temperature irregularities, respectively. However, the MAE expression avoids assuming a model for the soot absorption coefficient, but with the shortcoming of introducing an additional source of noise. In contrast, EMI expression provides smoother deconvolution fields, but requiring a spectral model for the soot absorption function. Note, also, that a value of $\alpha_{\text {reg }}=10^{-5}$ is sufficient to regularize the reconstruction fields of $J_{\lambda}$ not show here for the sake of brevity - but a value of $\alpha_{r e g}=10^{-4}$ seems to be excessively smoothing for the peak region of $k_{\lambda}$ and $J_{\lambda}$.

\subsection{Emission temperature fields}

The experimental soot temperature distributions obtained for two or three wavelengths combinations together with the maps of relative sensitive of temperature with respect to signal ratio are presented in Fig. 7. These experimental temperature fields have been determined resolving Eq. (3), neglecting the self-absorption exponential term, i.e., by considering the flame as being optically thin. The deconvolution and regularization procedure is applied to the CR only. An acceptable agreement may be observed for the soot temperature measured for different wavelength combinations (Figs. $7 \mathrm{a}, 7 \mathrm{~b}$ and $7 \mathrm{c}$ ). It is important to note that the similarity is reasonably good, upon comparison with the numerical temperature field given in Fig. 3b.

The discrepancy observed is notably reduced when the wavelengths pair is located at the infrared region, as is the case for the pair $\left(\lambda_{2}, \lambda_{3}\right)$, for instance. This 


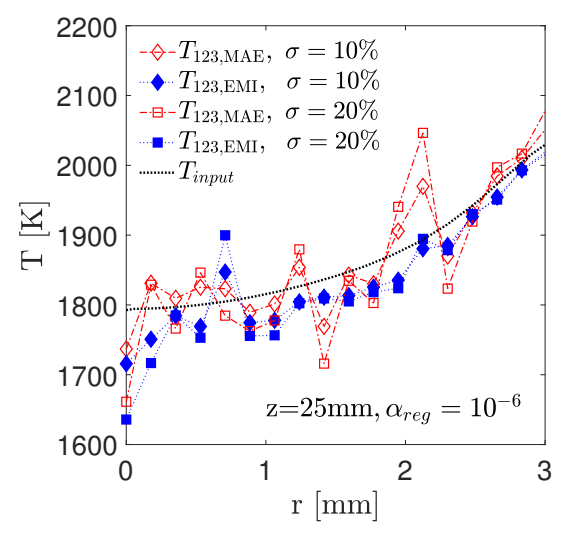

(a) $\alpha_{\text {reg }}=10^{-6}$

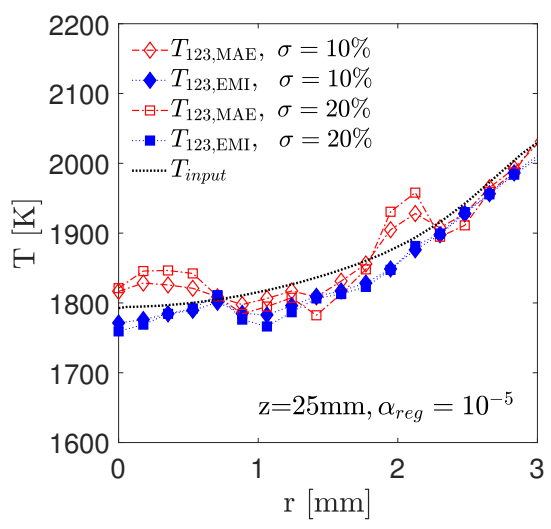

(b) $\alpha_{\text {reg }}=10^{-5}$

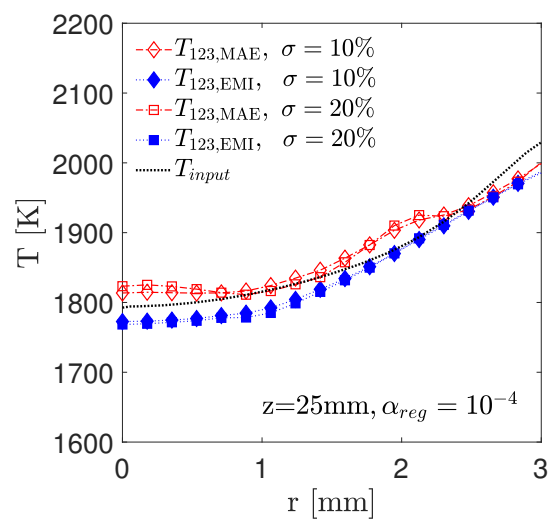

(c) $\alpha_{\text {reg }}=10^{-4}$

Figure 6: Influence of added uncorrelated white noise on the retrieved three color temperature, using different values for the regularization parameter $\alpha_{\text {reg }}$. Radial profiles at $z=25 \mathrm{~mm}$. 


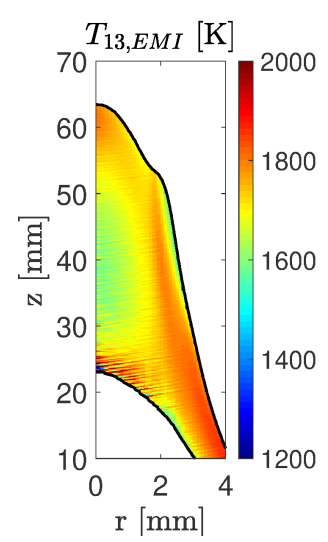

(a)

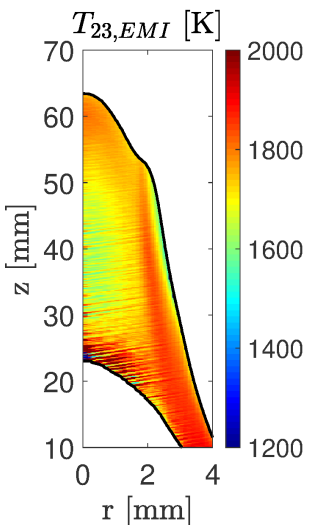

(b)

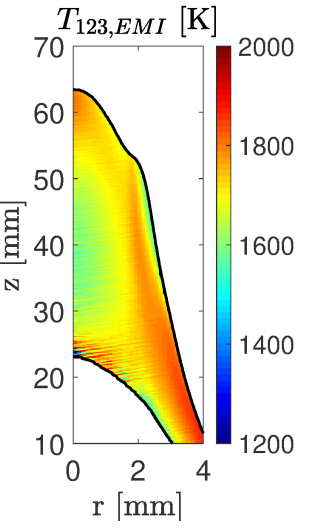

(c)

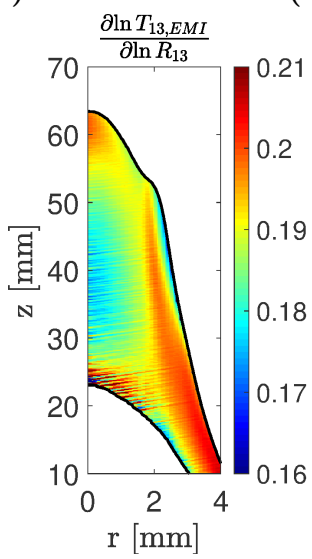

(d)

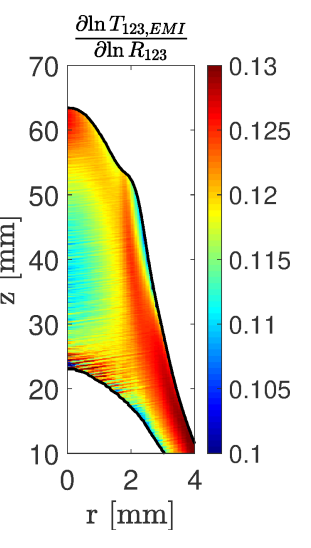

(e)

Figure 7: Temperature measured with the EMI two and three color methodology for an ethylene/air laminar coflow non-premixed flame (a) $T_{13, E M I}$, (b) $T_{23, E M I}$, (c) $T_{123, E M I}$, (d) relative error $\partial \ln T_{13, E M I} / \partial \ln R_{13}$ and (e) relative error $\partial \ln T_{123, E M I} / \partial \ln R_{123}$. 
is also underscored by the sensitivity analysis presented above and by previous experimental studies (Wang et al. 1996, Liu, Snelling, Thomson and Smallwood 2009). Additionally, this sensitivity analysis suggests that the three wavelengths model introduces a source of error associated to the measured intensity at the third wavelength. Indeed, the two wavelengths model is more sensitive than three wavelengths approach, as indicated by the fields of relative sensitivity of soot temperature to the intensity ratio given in Figs. $7 \mathrm{~d}$ and $7 \mathrm{e}$. From a physical point of view, it is desirable to have a small $\partial \ln T / \partial \ln R$ value, in order to prevent small changes (or errors) in the signal ratio, $R$, to cause a large change on the temperature estimate. Nevertheless, the best agreement with the numerical data has been obtained for the $T_{123}$ model. This could be associated to the used soot emission detection device. Currently the wavelengths employed are close to infrared region $\left(\lambda_{2}=660 \mathrm{~nm}\right.$ and $\left.\lambda_{3}=800 \mathrm{~nm}\right)$ and near the detection limit of the spectral response of the common CCD (or CMOS) camera. As a consequence, the camera noise could affect the integrated emission measured by introducing large errors. Therefore, the use of three wavelengths seems to be a good choice when the spectral detection is near to such limits.

\section{Conclusions}

A methodology to determine the soot temperature of axisymmetrical flames was presented, based on the natural soot emission measurement at different wavelengths. The radiative model employed was numerically verified using the integrated soot emission field, which was constructed from previously computed data of a target Gülder diffusion flame with an annular soot volume fraction distribution. The results showed a good agreement between the original and the retrieved temperature fields, when the self-absorption effect is either considered or neglected. The retrieved temperature results exhibited smaller differences within a confidence region that encompasses most of the sooting region. At this region boundary, which is characterized by small signal-to-noise ratios, the deconvolution procedure is compromised, and larger temperature discrepancies arise. Arbitrarily increasing the soot volume fraction yielded maximum temperature discrepancies smaller of $70 \mathrm{~K}$, even when the values of $f_{v}$ are doubled, thus underscoring the optically thin assumption correctness. However, neglecting the self-absorption effect leads to temperature discrepancies which arises mainly at the vicinity of the maximum soot volume fraction position. An analysis of the noise effect on the temperature showed that the MAE temperature calculation method is more sensitive than the EMI one. This increased sensitivity was shown to be associated to the noise contribution of the extinction field on the temperature reconstruction process and to the temperature equation terms, where a model of soot absorption function is required. If the required level of precision is smaller than $60 \mathrm{~K}$, the present emission methodology turns out to be an alternative to determine the flame temperature via a simplified experimental setup. Furthermore, measured temperature results at different combination of detection wavelengths showed a good distribution similarity. The performed 
sensitivity analysis indicated that temperature discrepancies could be reduced when the separation of wavelengths is increased. Also, the use of three wavelengths detection could be justified when the spectral response of the employed cameras within the infrared range are near their limits.

\section{Acknowledgments}

This work was supported by ANID Fondecyt/Postdoctoral 3190860 and ANID Fondecyt/Regular 1191758 grants. L.F. Figueira da Silva was on leave from the Institut Pprime (CNRS, France). The authors also gratefully acknowledge the support for the present research provided by Conselho Nacional de Desenvolvimento Científico e Tecnológico, CNPq, under the Research Grants No. 306069/2015-6 and 403904/2016-1 and from CAPES/PrInt grant 88887.310633/ 2018-00.

\section{References}

Åkesson, E. O. and Daun, K. J.: 2008, Parameter selection methods for axisymmetric flame tomography through tikhonov regularization, Appl. Opt. 47, 407-416.

Andrade, F. O., Figueira da Silva, L. F. and Mura, A.: 2011, Large eddy simulation of turbulent premixed combustion at moderate Damköhler numbers stabilized in a high-speed flow, Combust. Sci. Technol. 183, 645-664.

Araujo, A.: 2017, Multi-spectral pyrometry-a review, Meas. Sci. Technol. 28, 082002 .

Chang, H. and Charalampopoulos, T. T.: 1990, Determination of the wavelength dependence of refractive indices of flame soot, Proc. Roy. Soc. London A 430, 577-591.

Daun, K. J., Thomson, K. A., Liu, F. and Smallwood, G. J.: 2006, Deconvolution of axisymmetric flame properties using Tikhonov regularization, Appl. Opt. 45, 4638-4646.

De Iuliis, S., Barbini, M., Benecchi, S., Cignoli, F. and Zizak, G.: 1998, Determination of the soot volume fraction in an ethylene diffusion flame by multiwavelength analysis of soot radiation, Combust. Flame 115, 253-261.

Di Stasio, S. and Massoli, P.: 1994, Influence of the soot property uncertainties in temperature and volume-fraction measurements by two-colour pyrometry, Meas. Sci. Technol. 5, 1453.

Dobbins, R. A. and Megaridis, C. M.: 1991, Absorption and scattering of light by polydisperse aggregates, Appl. Opt. 30, 4747-4754. 
Eaves, N. A., Zhang, Q., Liu, F., Guo, H., Dworkin, S. B. and Thomson., M. J.: 2016, Coflame: A refined and validated numerical algorithm for modeling sooting laminar coflow diffusion flames, Comput. Phys. Commun. 207, 464477 .

Escudero, F., Fuentes, A., Consalvi, J.-L., Liu, F. and Demarco, R.: 2016, Unified behavior of soot production and radiative heat transfer in ethylene, propane and butane axisymmetric laminar diffusion flames at different oxygen indices, Fuel 183, 668-679.

Freeman, M. P. and Katz, S.: 1960, Determination of the radial distribution of brightness in a cylindrical luminous medium with self-absorption, J. Opt. Soc. Am. 50, 826-830.

Goulay, F., Schrader, P. E. and Michelsen, H. A.: 2010, Effect of the wavelength dependence of the emissivity on inferred soot temperatures measured by spectrally resolved laser-induced incandescence, Appl. Phys. B 100, 655-663.

Goulay, F., Schrader, P. E., Nemes, L., Dansson, M. A. and Michelsen, H. A.: 2009, Photochemical interferences for laser-induced incandescence of flamegenerated soot, Proc. Combust. Inst. 32, 963-970.

Greenberg, P. S. and Ku, J. C.: 1997, Soot volume fraction imaging, Appl. Opt. 36, 5514-5522.

Hadef, R., Geigle, K. P., Zerbs, J., Sawchuk, R. A. and Snelling, D. R.: 2013, The concept of $2 \mathrm{D}$ gated imaging for particle sizing in a laminar diffusion flame, Appl. Phys. B 112, 395-408.

Jakob, M., Lehnen, P., Adomeit, P. and Pischinger, S.: 2014, Development and application of a stereoscopic 3-color high-speed ratio-pyrometry, Combust. Flame 161, 2825-2841.

Jenkins, T. and Hanson, R.: 2001, Soot pyrometry using modulated absorption/emission, Combust. Flame 126, 1669-1679.

Jerez, A., Cruz Villanueva, J., Figueira da Silva, L., Demarco, R. and Fuentes, A.: 2019, Measurements and modeling of pah soot precursors in coflow ethylene/air laminar diffusion flames, Fuel 236, 452-460.

Kempema, N. J. and Long, M. B.: 2018, Effect of soot self-absorption on colorratio pyrometry in laminar coflow diffusion flames, Opt. Lett. 43, 1103-1106.

Khan, M. A., Allemand, C. and Eagar, T. W.: 1991, Noncontact temperature measurement. i. interpolation based techniques, Rev. Sci. Instrum. 62, 392402.

Legros, G., Wang, Q., Bonnety, J., Kashif, M., Morin, C., Consalvi, J.-L. and Liu, F.: 2015, Simultaneous soot temperature and volume fraction measurements in axis-symmetric flames by a two-dimensional modulated absorption/emission technique, Combust. Flame 162, 2705-2719. 
Li, M. and He, Z.: 2019, Research on the effect of soot self-absorption on flame multispectral radiation reconstruction, J. Thermophys. Heat Trans. 33, 179188.

Liu, F., Snelling, D. R., Thomson, K. and Smallwood, G. J.: 2009, Sensitivity and relative error analyses of soot temperature and volume fraction determined by two-color LII, Appl. Phys. B 96, 623-636.

Liu, F., Thomson, K. A. and Smallwood, G. J.: 2009, Numerical investigation of the effect of signal trapping on soot measurements using LII in laminar coflow diffusion flames, Appl. Phys. B 96, 671-682.

Liu, F., Thomson, K. A. and Smallwood, G. J.: 2013, Soot temperature and volume fraction retrieval from spectrally resolved flame emission measurement in laminar axisymmetric coflow diffusion flames: Effect of self-absorption, Combust. Flame 160, 1693-1705.

Michelsen, H. A., Schrader, P. E. and Goulay, F.: 2010, Wavelength and temperature dependences of the absorption and scattering cross sections of soot, Carbon 48, 2175-2191.

Migliorini, F., Thomson, K. A. and Smallwood, G. J.: 2011, Investigation of optical properties of aging soot, Appl. Phys. B 104, 273-283.

Modest, M. F.: 2013, Radiative Heat Transfer, third edition edn, Academic Press, Boston.

Panagiotou, T., Levendis, Y. and Delichatsios, M.: 1996, Measurements of particle flame temperatures using three-color optical pyrometry, Combust. Flame 104, 272-287.

Patiño, F., Cruz, J., Verdugo, I., Morn, J., Consalvi, J., Liu, F., Du, X. and Fuentes, A.: 2020, Soot primary particle sizing in a n-heptane doped methane/air laminar coflow diffusion flame by planar two-color tire-lii and tem image analysis, Fuel 266, 117030.

Snelling, D. R., Thomson, K. A., Smallwood, G. J. and Gülder, O. L.: 1999, Two-dimensional imaging of soot volume fraction in laminar diffusion flames, Appl. Opt. 38, 2478-2485.

Snelling, D. R., Thomson, K. A., Smallwood, G. J., Gülder, O. L., Weckman, E. J. and Fraser, R. A.: 2002, Spectrally resolved measurement of flame radiation to determine soot temperature and concentration, AIAA Journal 40, 1789-1795.

Vedovoto, J., Silveira Neto, A., Figueira da Silva, L. F. and Mura, L.: 2015, Influence of synthetic inlet turbulence on the prediction of low Mach number flows, Comput. Fluids. 106, 135-153. 
Viskanta, R. and Mengüç, M. P.: 1987, Radiation heat transfer in combustion systems, Prog. Energ. Combust. Sci. 13, 97-160.

Wang, H.: 2011, Formation of nascent soot and other condensed-phase materials in flames, Proc. Combust. Inst. 33, 41-67.

Wang, Q., Legros, G., Morin, C., Yao, M., Cai, W. and Jiang, L.: 2019, Optical measurements of temperature fields in sooting flames: influence of soot selfabsorption, Appl. Phys. B $\mathbf{1 2 5}$.

Wang, Y., Yao, M. and Liao, Y.: 1996, Selection of optimal working wavelengths and bandwidths for dual-wavelength optical fiber pyrometer, Proc. of the SPIE 2594, 75-80.

Yon, J., Lemaire, R., Therssen, E., Desgroux, P., Coppalle, A. and Ren, K.: 2011, Examination of wavelength dependent soot optical properties of diesel and diesel/rapeseed methyl ester mixture by extinction spectra analysis and lii measurements, Appl. Phys. B 104, 253-271.

Zhao, H. and Ladommatos, N.: 1998, Optical diagnostics for soot and temperature measurement in diesel engines, Prog. Energ. Combust. Sci. 24, 221-255.

Zhou, B., Costa, M., Li, Z., Aldén, M. and Bai, X.-S.: 2017, Characterization of the reaction zone structures in a laboratory combustor using optical diagnostics: from flame to flameless combustion, Proc. Combust. Inst. 36, 4305-4312. 